\title{
NERVOUS DISEASES.
}

BY DR. G. S. MIDDLETON.

Periodic Paraplegia.-Professor Fischl, of Prague, published in the Prag. Medizin. Wochenschr., 21st October 1885, a case of temporary and periodic paraplegia. The patient, 8 years of age, with no particular hereditary tendency, had an attack of scarlatina, followed by nephritis. She had been entirely cured of this affection for several months, when she was seized with a feeling of general weariness, headache, backache, and progressive loss of appetite. For several days she had also great drowsiness. In the midst of those symptoms there occurred one morning, without loss of consciousness, a complete paralysis of the legs, and a temporary feebleness of the arms. The latter disappeared directly. The paraplegia, on the contrary, lasted three hours, to disappear completely directly thereafter. This paraplegia was accompanied by a complete absence of sensibility, and by a notable lowering of the surface temperature, up to several fingers' breadths above the knees; the abdominal reflex was preserved; the knee-jerk was abolished; the plantar reflex was elicited only by deep pricking; the muscles reacted in no way. There was nothing to note in the thoracic or abdominal organs.

Four days later the same disorders were repeated, and were evolved in the same way. During this attack examination of the muscles by the Faradic current showed a complete absence of reaction in the tibial and peroneal muscles, and an irregular action in the domain of the crural nerve. During six days there was nothing to note; and then occurred a new attack, lasting only half an hour, followed, three days later, by a fourth attack still less pronounced, but involving also the upper limbs, and here again the paralysis of the upper limbs was the first to disappear.

These attacks of paraplegia were later followed by paroxysms of cerebral symptoms, analogous to those which preceded the first attack, consisting of a feeling of fatigue suddenly giving place to sleep for half an hour. Complete recovery then took place.

Comparable to the above is the case, published by Westphal (Ertl. Intell. Blatt, No. 44, 1885), of a boy of 12 years, attacked with paralysis recurring at intervals of one to four weeks, and persisting only for a few hours. In each attack there was observed paralysis first of the lower limbs, then 
spreading to the muscles of the neck and of the upper limbs, and generally becoming complete during the night, without the least trace of contraction or of muscular rigidity. There was complete absence of disturbance of sensibility, and of the muscles of the head, face, and eyes. On a single occasion there was retention of urine. There was loss of the plantar reflex, and of the knee-jerk, while the cremasteric and abdominal reflexes were intact. On a single occasion also there was a slight persistence of the paralysis in the front of the legs. A most remarkable fact was the complete absence of reaction to the Faradic and galvanic currents on the part of all the muscles attacked by the paralysis.

Westphal draws attention to the length of the intervals between the attacks of paralysis, and to their irregularity. This prevents us from classing this case among those referred to the malarial influence; besides, no observations on "intermittent" paralysis mention the absolute loss and the early return of the electric reaction. Westphal can offer no etiological interpretation of the facts above recorded.-Rev. Internat. des Sci. Méd., 31st January, 1886.

Acute Anterior Poliomyelitis.-Dr. R. A. Gibbons records, under the above title, a most interesting case of a boy, aged 9 years, whose symptoms were referred to a blow on the lower back, seven days after which they commenced with pain in the thighs, followed by paralysis, which involved both lower extremities, and partially the arms. Neither sensation, nor the bowels, nor bladder were affected; but the reflexes, both superficial and deep, were gradually lost, and the muscles entirely failed to respond to the Faradic current. There was slight fever. The course of the disease was very interesting. From the commencement of symptoms to the stage of absolute paralysis was forty-eight hours: and from the onset of paralysis to recovery of reflexes and return of response to Faradic current was seven days. The treatment mainly was by tincture of belladonna in ten minim doses every four hours. The result was perfect recovery. Dr. Gibbons discusses very fully the probable pathology of this case, which, it must be admitted, is not free from difficulty.-Med. Times and Gazette, 5th Sept., 1885.

Paralysis with Atrophy of the Supra- and InfraSpinatus Muscles.-Six months before being seen by M. Nicaise the patient had received a severe blow in the right supra-spinous fossa, and had been unable to work since. 
There was, at first, pain, after the disappearance of which the arm remained powerless. When seen there was marked atrophy limited to the supra- and infra- spinatus muscles, and, although the movements of the forearm could be executed, he could not, for example, lift a glass. The rhomboid, trapezius, serratus magnus, deltoid, and all the muscles of the arm were intact. The paralysis in this case was due to the fact that the head of the humerus could not be properly kept in apposition with the glenoid facet, which is the function of the supra- and infra- spinatus and other muscles. Although only one of the muscles was struck, both were affected, and $\mathbf{M}$. Nicaise infers that probably a branch of the supra-scapular nerve was contused, giving rise to a consecutive neuritis of the whole nerve. By Faradisation the patient was cured.-La France Médicale, 7th March, 1885.

Cases of Ophthalmoplegia, complicated with various other affections of the Nervous System.-Dr. Bristowe reports a remarkably interesting series of cases with the above title in Brain (Oct., 1885), the summaries of which may be given briefly in his own words :-

"Case I. Graves' disease, followed by ophthalmoplegia externa, right hemianæsthesia, with involvement of organs of special sense, headache, sickness, and persistent high temperature, and subsequently by right hemiplegia, epileptic fits, bleeding from the ears, \&c.-Death from bronchitis."

"Case II. Ophthalmoplegia externa, right hemiplegia, headache, and sickness, followed by partial right hemianæsthesia, and epileptic fits, preceded by prolonged rises of temperature. Chorea during progress of patient's illness.-No result."

There was a singularly close resemblance between those cases. In both there was almost complete ophthalmoplegia externa, paralysis with rigidity of the right arm and leg, and more or less complete right hemianæsthesia; both patients suffered severely from headache and sickness, and frequent fits of an epileptiform character. In the first case there was constant bleeding from the ears (for very many months); and there were also colour-blindness, and loss of taste and smell on the right side; all of which were wanting in the second case, in which there was paralysis of the right side of the tongue, which was not observed in the other. In both cases there was frequent febrile rise of temperature, but in the first the elevation of temperature was more or less persistent, and had no apparent relation to anything in particular, while in the second it was comparatively rarely present except as the 
forerunner of epileptic fits, after which it suddenly fell to normal.

The diagnosis formed of the first ease was some degenerative change or a tumour occupying the floor of the anterior part of the fourth ventricle and the walls of the iter, with extension into the neighbouring sensory tract on the left side, and probably also a growth involving the dura mater in the neighbourhood of the petrous bones, and possibly the petrous bones themselves. As the case had been under observation for several years, and had given rise to a great deal of interest, the post-mortem examination was looked forward to with extreme interest. But the most diligent search, naked eye and microscopic, failed to discover any trace of disease in the brain or cord, or in any of the intra-cranial tissues, and the hæmorrhage from the ears remained unaccounted for.

From the results of the post-mortem examination, Dr. Bristowe states that the disease must be looked upon as functional, but he declines to regard it as hysterical. He infers from it that the second case may also be functional, and he further argues that many cases of obscure and progressive brain disease which do not seem to be hysterical, and which simulate organic disease, may prove amenable to treatment or to the influence of time.

"Case III. Ophthalmoplegia externa and interna; partial anæsthesia of head and neck and chest; epileptic fits; gastric crises; and attacks of intense dyspnœea dependent on paralysis of the abductors of the vocal cords."

"Case IV. Ophthalmoplegia externa ; wasting palsy."

"Case V. Ophthalmoplegia externa and interna; locomotor ataxy, attended with gastric and rectal distress, and a peculiar cough."

\section{DISEASES OF THE EAR.}

By Dr. WALKER DOWNIE.

Aural Disease in Diabetes Mellitus. (Deutsche Med. Wochenschrift, 24th December, 1885).-Of late years, in a few instances, an attempt has been made to trace an etiological connection between a form of acute otitis media, and diabetes mellitus, in which disease there certainly does occur now and then an acute inflammation of the middle ear and mastoid process, not dependent upon an external cause, so far as can be discovered by the most careful search.

Schwabach, in a paper on this subject in Journal above 